\title{
Nef cone volumes and discriminants of abelian surfaces
}

\author{
Thomas Bauer, Carsten Bornträger
}

Version of August 25, 2016

\begin{abstract}
The nef cone volume appeared first in work of Peyre in a number-theoretic context on Del Pezzo surfaces, and it was studied by Derenthal and co-authors in a series of papers. The idea was subsequently extended to also measure the Zariski chambers of Del Pezzo surfaces. We start in this paper to explore the possibility to use this attractive concept to effectively measure the size of the nef cone on algebraic surfaces in general. This provides an interesting way of measuring in how big a space an ample line bundle can be moved without destroying its positivity. We give here complete results for simple abelian surfaces that admit a principal polarization and for products of elliptic curves.
\end{abstract}

\section{Introduction}

The concept of nef cone volume appears first in work of Peyre [9] in a numbertheoretic context (Manin's conjecture) on Del Pezzo surfaces, and was subsequently studied by Derenthal and co-authors in a series of papers [3, 4, 5, 6, 7, 8]. It was shown in [1] that an extension of this notion can be used, beyond its numbertheoretical origins, to measure the size of Zariski chambers (and also of the whole big cone) on Del Pezzo surfaces. In the present paper we start exploring the possibility to use this attractive concept to effectively measure the size of the nef cone (or the big cone) on algebraic surfaces in general.

To begin with, it is natural to generalize the construction from [5] by fixing an ample line bundle $H$ on $X$ (which was $-K_{X}$ in the Del Pezzo case) and proceeding in the following way: Using the half-space

$$
H^{\leqslant 1}=\left\{L \in \mathrm{NS}_{\mathbb{R}}(X) \mid H \cdot L \leqslant 1\right\}
$$

we define for any cone $\mathcal{C} \subset \mathrm{NS}_{\mathbb{R}}(X)$ its cone volume with respect to $H$ as the volume of the truncated cone $\mathcal{C} \cap H^{\leqslant 1}$. We will use the notation

$$
\operatorname{Vol}(\mathcal{C}, H):=\operatorname{Vol}\left(\mathcal{C} \cap H^{\leqslant 1}\right) .
$$

Here the volume on the right-hand side is taken after identifying the Néron-Severi vector space $\mathrm{NS}_{\mathbb{R}}(X)$ with $\mathbb{R}^{\rho(X)}$ by means of an isomorphism induced by a lattice basis. (In other words, we normalize the volume on $\mathrm{NS}_{\mathbb{R}}(X)$ by requiring that a fundamental parallelotope of the lattice $\operatorname{NS}(X)$ has volume one). The quantity is

Keywords: abelian surface, ample line bundle, nef cone, volume, discriminant.

Mathematics Subject Classification (2010): 14C20, $14 \mathrm{~K} 05$. 
then independent of the choice of the lattice basis. In particular, the nef cone volume of $X$ with respect to $H$,

$$
\operatorname{Vol}(\operatorname{Nef}(X), H),
$$

is then an invariant that is naturally attached to the polarized surface $(X, H)$. Geometrically, we may think of it as telling us in how big a space an ample line bundle can be moved without destroying its positivity.

For simple principally polarized abelian surfaces and for products of two elliptic curves, we develop in this paper a complete picture of how nef cone volumes are behaved.

Theorem 1 Let $X$ be a simple abelian surface which admits a principal polarization, and let $H$ be any ample line bundle on $X$ (not necessarily a multiple of the principal polarization). Then the nef cone volume of $X$ with respect to $H$ can be determined in terms of $\operatorname{End}(X)$, specifically:

(a) Suppose that $X$ has only integer multiplication, i.e., $\operatorname{End}(X)=\mathbb{Z}$. Then

$$
\operatorname{Vol}(\operatorname{Nef}(X), H)=\frac{1}{\sqrt{2 H^{2}}} .
$$

(b) Suppose that $X$ has real multiplication, i.e., $\operatorname{End}_{\mathbb{Q}}(X)=\mathbb{Q}(\sqrt{d})$ for some square-free integer $d>0$, and let $f \geqslant 1$ be the conductor of $\operatorname{End}(X)$ in $\operatorname{End}_{\mathbb{Q}}(X)$ (see Sect. $\mathbb{Q}$ for details). Then

$$
\operatorname{Vol}(\operatorname{Nef}(X), H)= \begin{cases}\frac{1}{2 f \sqrt{d}\left(H^{2}\right)} & \text { if } d \equiv 2,3(\bmod 4) \\ \frac{1}{f \sqrt{d}\left(H^{2}\right)} & \text { if } d \equiv 1(\bmod 4)\end{cases}
$$

The same formula applies when $X$ has complex multiplication: In that case the numbers $f$ and $d$ are to be taken from the order $\operatorname{End}^{\mathrm{sym}}(X)$ in the realquadratic subfield $\operatorname{End}_{\mathbb{Q}}^{\mathrm{sym}}(X)=\mathbb{Q}(\sqrt{d}) \subset \operatorname{End}_{\mathbb{Q}}(X)$.

(c) Suppose that $X$ has indefinite quaternion multiplication, and write $\operatorname{End}_{\mathbb{Q}}(X)=$ $\mathbb{Q}+i \mathbb{Q}+j \mathbb{Q}+i j \mathbb{Q}$, and $\operatorname{End}(X)=\mathbb{Z} \oplus \mathbb{Z} a \oplus \mathbb{Z} b \oplus \mathbb{Z} a b$ with suitable primitive Rosati invariant elements. Then

$$
\operatorname{Vol}(\operatorname{Nef}(X), H)=\frac{\pi \sqrt{2}}{3 \sqrt{\left|\operatorname{det} S_{\delta}(a, b)\right|}\left(H^{2}\right)^{\frac{3}{2}}} .
$$

(see Sect. 2 for details, in particular for the definition of the matrix $S_{\delta}(a, b)$.)

Theorem 2 Let $X=E_{1} \times E_{2}$ be a product of two elliptic curves, and let $H$ be any ample line bundle on $X$.

(a) If $E_{1}$ and $E_{2}$ are not isogenous, then

$$
\operatorname{Vol}(\operatorname{Nef}(X), H)=\frac{1}{\left(H^{2}\right)}
$$


(b) Suppose that $E_{1}$ and $E_{2}$ are isogenous and have no complex multiplication, i.e. $\operatorname{End}\left(E_{1}\right)=\operatorname{End}\left(E_{2}\right)=\mathbb{Z}$. If $\sigma: E_{1} \rightarrow E_{2}$ is an isogeny of minimal degree, then

$$
\operatorname{Vol}(\operatorname{Nef}(X), H)=\frac{\pi}{3 \cdot \sqrt{2 \cdot \operatorname{deg}(\sigma)} \cdot\left(H^{2}\right)^{\frac{3}{2}}} .
$$

(c) Suppose that $E_{1}$ and $E_{2}$ are isogenous and have complex multiplication. Write $\operatorname{End}_{\mathbb{Q}}\left(E_{i}\right)=\mathbb{Q}(\sqrt{d})$ with a square-free integer $d<0$, and let $f_{1}$ and $f_{2}$ the conductors of $\operatorname{End}\left(E_{1}\right)$ and $\operatorname{End}\left(E_{2}\right)$, respectively. Then

$$
\operatorname{Vol}(\operatorname{Nef}(X), H)= \begin{cases}\frac{\pi}{6 \cdot \operatorname{LCM}\left(f_{1}, f_{2}\right) \sqrt{|d|}\left(H^{2}\right)^{2}} & \text { if } d \equiv 2,3(\bmod 4) \\ \frac{\pi}{3 \cdot \operatorname{LCM}\left(f_{1}, f_{2}\right) \sqrt{|d|}\left(H^{2}\right)^{2}} & \text { if } d \equiv 1(\bmod 4) .\end{cases}
$$

Our work is based on the observation that the volume of the positive cone

$$
\operatorname{Pos}(X)=\left\{D \in \mathrm{NS}_{\mathbb{R}}(X) \mid D^{2} \geqslant 0, H \cdot D \geqslant 0\right\}
$$

is governed (on any smooth projective surface) by the discriminant of the NéronSeveri lattice (see Prop 1.11). In the case of abelian surfaces, the positive cone and the pseudo-effective cone both coincide with the nef cone. Therefore, the issue in determining the nef cone volume is to find effective ways to determine the discriminant. We show for simple abelian surfaces how the discriminant depends on the structure of the endomorphism ring and we determine it in terms of the ring-theoretic data (see Section 2). For products of elliptic curves $E_{1} \times E_{2}$, the main case is when $E_{1}$ and $E_{2}$ are isogenous curves with complex multiplication; the crucial point here is to express the discriminant in terms of the conductors (see Theorem 3.5).

Note that, as we make essential use of the isomorphism of $\operatorname{NS}(X)$ with the group $\operatorname{End}^{\mathrm{sym}}(X)$ of symmetric endomorphisms, our present methods shed no light on the non-principally polarized case. The question of nef cone volumes is of course equally of interest there, and it would be very interesting to see how the picture extends.

\section{The volume of the positive cone on algebraic surfaces}

The positive cone of a smooth projective surface $X$ is by definition

$$
\operatorname{Pos}(X)=\left\{D \in \mathrm{NS}_{\mathbb{R}}(X) \mid D^{2} \geqslant 0, H \cdot D \geqslant 0\right\}
$$

where $H$ is a fixed ample divisor. We show here that its cone volume can be computed in terms of the self-intersection of $H$ and the discriminant of the Néron-Severi lattice:

Proposition 1.1 Let $X$ be a smooth projective surface and $H$ an ample divisor. Denote by $\rho$ the Picard number of $X$ and by $\Delta$ the discriminant of the Néron-Severi group (i.e., the determinant of the Gram matrix with respect to a lattice basis of $\mathrm{NS}(X)$ ). Then the cone volume of the positive cone is given by the formula

$$
\operatorname{Vol}(\operatorname{Pos}(X), H)=\frac{V_{\rho}}{\sqrt{|\Delta|} \cdot\left(H^{2}\right)^{\rho / 2}}
$$


where $V_{\rho}$ is the volume of the truncated cone

$$
\left\{x \in \mathbb{R}^{\rho} \mid 0 \leqslant x_{1} \leqslant 1 \text { and } x_{1}^{2}-x_{2}^{2}-\cdots-x_{\rho}^{2} \geqslant 0\right\} .
$$

Remark 1.2 For abelian surfaces we have $1 \leqslant \rho \leqslant 4$, so in that case we will only need the following numerical values of $V_{\rho}$ :

$$
V_{1}=V_{2}=1, \quad V_{3}=V_{4}=\frac{\pi}{3}
$$

Proof of the proposition. Let $S$ be the matrix of the intersection form with respect to a lattice basis. We can find an $\mathbb{R}$-basis $B_{1}, \ldots, B_{\rho}$ of $\mathrm{NS}_{\mathbb{R}}(X)$ with respect to which the intersection form is diagonal and where $B_{1}=1 / \sqrt{H^{2}} \cdot H$. Let $T \in \operatorname{GL}(n, \mathbb{R})$ be a change of base matrix satisfying $T^{t} S T=\operatorname{Diag}(1,-1, \ldots,-1)$. In particular, we have then $|\operatorname{det} T|=1 / \sqrt{|\Delta|}$, as $\Delta=\operatorname{det} S$. The truncated positive cone

$$
\operatorname{Pos}(X) \cap H^{\leqslant 1}
$$

(more precisely, its coordinate set with respect to the lattice basis) is mapped by $T^{-1}$ to the set

$$
\left\{x \in \mathbb{R}^{\rho} \mid 0 \leqslant x_{1} \leqslant \frac{1}{\sqrt{H^{2}}} \text { and } x_{1}^{2}-x_{2}^{2}-\cdots-x_{\rho}^{2} \geqslant 0\right\}
$$

Upon multiplying this set by $\sqrt{H^{2}}$, we obtain the subset of $\mathbb{R}^{\rho}$ given in the statement of the proposition. Therefore the volume of the latter set is given by

$$
V_{\rho}=\left(\sqrt{H^{2}}\right)^{\rho} \cdot\left|\operatorname{det} T^{-1}\right| \cdot \operatorname{Vol}\left(\operatorname{Pos}(X) \cap H^{\leqslant 1}\right)
$$

and this implies the assertion.

Thanks to the inclusions of cones

$$
\operatorname{Nef}(X) \subset \operatorname{Pos}(X) \subset \overline{\operatorname{Big}}(X)
$$

the proposition immediately yields the following estimates:

Corollary 1.3 In the situation of Prop 1.1 we have

$$
\operatorname{Vol}(\operatorname{Nef}(X), H) \leqslant \frac{V_{\rho}}{\sqrt{|\Delta|} \cdot\left(H^{2}\right)^{\rho / 2}} \leqslant \operatorname{Vol}(\overline{\operatorname{Big}}(X), H) .
$$

This shows in particular that the nef cone volume can become arbitrarily small since $V_{\rho} / \sqrt{|\Delta|}$ can be arbitrarily small. (This happens for suitable abelian surfaces with real multiplication, see Sect. 2.)

On abelian surfaces, the positive cone and the pseudo-effective cone both coincide with the nef cone. Therefore, as a consequence of Prop. 1.1, determining nef cone volumes of abelian surfaces is equivalent to determining their discriminants:

Corollary 1.4 Let $(X, H)$ be a polarized abelian surface. Then the nef cone volume of $X$ is given by

$$
\operatorname{Vol}(\operatorname{Nef}(X), H)=\frac{V_{\rho}}{\sqrt{|\Delta|} \cdot\left(H^{2}\right)^{\rho(X) / 2}} .
$$




\section{Volumes of simple abelian surfaces}

Let in this section $X$ be a simple abelian surface admitting a principal polarization $L_{0}$. Our purpose it to determine the nef cone volume

$$
\operatorname{Vol}(\operatorname{Nef}(X), H)
$$

with respect to any ample line bundle $H$ on $X$. We will make use of the classification of endomorphism algebras of abelian varieties (see [2]), and work according to whether $X$ has integer, real, complex or quaternion multiplication. The results in this section prove Theorem 1 from the introduction.

Type 0: Integer multiplication. Suppose that $\operatorname{End}(X)=\mathbb{Z}$. As $L_{0}$ gives a basis of $\operatorname{NS}(X)$, we have $\Delta(X)=2$ for the discriminant. Prop 1.1 then implies

$$
\operatorname{Vol}(\operatorname{Nef}(X), H)=\frac{1}{\sqrt{2} \cdot \sqrt{H^{2}}} .
$$

Alternatively, the result can in this simple case be obtained more directly by noting that for $H=c \cdot L_{0}$ with $c>0$ we have:

$$
H^{\leqslant 1}=\{L \in \operatorname{Nef}(X) \mid H \cdot L \leqslant 1\} \simeq\left\{x \in \mathbb{R} \mid x \leqslant \frac{1}{2 c}\right\}
$$

and hence the truncated nef cone is

$$
\operatorname{Nef}(X) \cap H^{\leqslant 1} \simeq\left[0, \frac{1}{2 c}\right]
$$

which has volume $\frac{1}{2 c}=1 /\left(\sqrt{2} \cdot \sqrt{H^{2}}\right)$.

Type 1: Real multiplication. Suppose that $X$ has real multiplication, i.e., that $\operatorname{End}_{\mathbb{Q}}(X)=\mathbb{Q}(\sqrt{d})$ for some square-free integer $d>0$. The endomorphism ring is an order in $\operatorname{End}_{\mathbb{Q}}(X)$, and hence of the form $\operatorname{End}(X)=\mathbb{Z}+f \omega \mathbb{Z}$, where $f \geqslant 1$ is an integer and

$$
\omega= \begin{cases}\sqrt{d} & \text { if } d \equiv 2,3(\bmod 4) \\ \frac{1}{2}(1+\sqrt{d}) & \text { if } d \equiv 1(\bmod 4)\end{cases}
$$

The isomorphism of groups

$$
\varphi: \operatorname{NS}(X) \rightarrow \operatorname{End}(X), \quad L \mapsto \phi_{L_{0}}^{-1} \phi_{L}
$$

provides us with a lattice basis of $\mathrm{NS}(X)$, given by $L_{0}=\varphi^{-1}(1)$ and $L_{f \omega}:=\varphi^{-1}(f \omega)$. The intersection matrix of this basis is

$$
\left(\begin{array}{cc}
2 & 0 \\
0 & -2 f^{2} d
\end{array}\right) \quad \text { if } d \equiv 2,3(\bmod 4)
$$

and

$$
\left(\begin{array}{cc}
2 & f \\
f & \frac{1}{2} f^{2}(1-d)
\end{array}\right) \quad \text { if } d \equiv 1(\bmod 4)
$$

This follows by considering the characteristic polynomial of $f \omega$ in $\mathbb{Q}(\sqrt{d})$ (which coincides with the analytic characteristic polynomial of the endomorphism) and applying [2, Prop. 5.2.3]. Therefore the discriminant is given by

$$
\Delta(X)=-4 f^{2} d \quad \text { and } \quad \Delta(X)=-f^{2} d,
$$


respectively. So by Prop. 1.1 we obtain for any ample line bundle $H$

$$
\operatorname{Vol}(\operatorname{Nef}(X), H)= \begin{cases}\frac{1}{2 f \sqrt{d}\left(H^{2}\right)} & \text { if } d \equiv 2,3(\bmod 4) \\ \frac{1}{f \sqrt{d}\left(H^{2}\right)} & \text { if } d \equiv 1(\bmod 4)\end{cases}
$$

The formula shows in particular that if we increase the "size" of the real multiplication $\sqrt{d}$, then the nef cone gets smaller at the rate of $1 / \sqrt{d}$. If we increase $f$ (thus making $\operatorname{End}(X)$ smaller), then the volume decreases at the rate of $1 / f$. This shows that nef cone volume can become arbitrarily small.

Type 2: Complex multiplication. Suppose that $X$ has complex multiplication, i.e., that $\operatorname{End}_{\mathbb{Q}}(X)$ is isomorphic to an imaginary quadratic extension of a real quadratic number field.

We have $\operatorname{End}_{\mathbb{Q}}^{\text {sym }}(X)=\mathbb{Q}(\sqrt{d})$, where $d$ is a positive square-free integer. Also $\operatorname{End}^{\mathrm{sym}}(X)$ has to be an order in $\operatorname{End}_{\mathbb{O}}^{\text {sym }}(X)$, and hence the arguments given for Type 1 apply here as well, when applied to the real-quadratic subfield $\operatorname{End}_{\mathbb{Q}}^{\text {sym }}(X)$.

Type 3: Indefinite quaternion multiplication. Suppose now that $X$ has indefinite quaternion multiplication, i.e., there are $\alpha, \beta \in \mathbb{Z} \backslash\{0\}$ with $\alpha \geqslant \beta$ and $\alpha>0$ such that $\operatorname{End}_{\mathbb{Q}}(X)=\mathbb{Q}+i \mathbb{Q}+j \mathbb{Q}+i j \mathbb{Q}$, where $i$ and $j$ satisfy the relations $i^{2}=\alpha, j^{2}=\beta$ and $i j=-j i$.

By [10, Theorem 7] we have $\operatorname{End}(X)=\mathbb{Z} \oplus \mathbb{Z} a \oplus \mathbb{Z} b \oplus \mathbb{Z} a b$, for some primitive Rosati invariant elements $a$ and $b$. In a rational quaternion algebra any element $x$ satisfies the equation $x^{2}-t(x) x+n(x)=0$, where $t(x)$ and $n(x)$ are the reduced trace and norm. Since the analytic representation $\rho_{\text {an }}: \operatorname{End}_{\mathbb{Q}}(X) \rightarrow M_{2}(\mathbb{C})$ is a ring homomorphism, the matrix $\rho_{\text {an }}(a)$ is a root of $x^{2}-t(a) x+n(a)$ and therefore the characteristic polynomial of $\rho_{\mathrm{an}}(a)$ coincides with $x^{2}-t(a) x+n(a)$. The same applies to $b$. Via the isomorphism

$$
\varphi: \mathrm{NS}(X) \rightarrow \operatorname{End}^{\mathrm{sym}}(X)=\mathbb{Z} \oplus \mathbb{Z} a \oplus \mathbb{Z} b, \quad L \mapsto \phi_{L_{0}}^{-1} \phi_{L}
$$

we get a basis

$$
L_{0}=\varphi^{-1}(1), L_{a}=\varphi^{-1}(a), L_{b}=\varphi^{-1}(b)
$$

of $\mathrm{NS}(X)$ and by [2, Prop. 5.2.3] we get their intersection matrix

$$
\left(\begin{array}{ccc}
2 & t(a) & t(b) \\
t(a) & 2 n(a) & n(a+b)-n(a)-n(b) \\
t(b) & n(a+b)-n(a)-n(b) & 2 n(b)
\end{array}\right)
$$

The determinant of the matrix above turns out to be the half of the determinant of the discriminant matrix

$$
S_{\delta}(a, b)=\left(\begin{array}{cc}
\delta(a) & \delta(a, b) \\
\delta(a, b) & \delta(b)
\end{array}\right)
$$

where the (quaternion) discriminant form is given by $\delta(x, y)=t(x) t(y)-2 n(x, y)$ with $n(x, y)=n(x+y)-n(x)-n(y)$. So we have

$$
\Delta(X)=\frac{1}{2} \operatorname{det}\left(S_{\delta}(a, b)\right)
$$


and therefore, we get by Proposition 1.1 for any ample line bundle $H$ :

$$
\operatorname{Vol}(\operatorname{Nef}(X), H)=\frac{\pi \sqrt{2}}{3 \sqrt{\left|\operatorname{det} S_{\delta}(a, b)\right|}\left(H^{2}\right)^{\frac{3}{2}}} .
$$

Remark 2.1 (Non-principally polarized case) It would be very interesting to see how the picture extends to the non-principally polarized case. A natural idea is to try using the fact that every polarization is the pullback of a principal polarization: Given any polarized abelian surface $(X, L)$, there is an isogeny

$$
f: X \rightarrow X_{0}
$$

where $X_{0}$ is an abelian surface carrying a principal polarization $L_{0}$ with $L=f^{*} L_{0}$. One might then hope to relate the discriminants $\Delta(X)$ and $\Delta\left(X_{0}\right)$ via the isogeny (e.g. its degree). However, it seems that there is no simple relation of this kind - to illustrate this, consider the following two situations:

- Suppose that $L$ generates $\operatorname{NS}(X)$ and is of type $(1, d)$. (This case is in reality uninteresting from a volume perspective because of $\rho(X)=1$.) So $f$ is of degree $d$. In this case,

$$
\Delta(X)=d \cdot \Delta\left(X_{0}\right) .
$$

- Suppose that $X$ is a product $E_{1} \times E_{2}$ of non-isogenous elliptic curves. There are isogenies $E_{1} \times E_{2} \rightarrow E_{1} \times E_{2}$ of arbitrarily high degree, but the discriminants $\Delta\left(E_{1} \times E_{2}\right)$ and $\Delta\left(E_{1}^{\prime} \times E_{2}^{\prime}\right)$ are both 2, independently of the chosen isogeny.

\section{Volumes of products of elliptic curves}

In this section we consider products $X=E_{1} \times E_{2}$ of elliptic curves. We will determine the nef cone volume $\operatorname{Vol}(\operatorname{Nef}(X), H)$ with respect to any ample line bundle $H$ on $X$. We will work according to whether $E_{1}$ and $E_{2}$ are isogenous, and whether they have complex multiplication - this amounts to three types A,B,C below. The results in this section prove Theorem 2 from the introduction.

To begin with, recall that we have

$$
\operatorname{End}(X)=\left(\begin{array}{cc}
\operatorname{End}\left(E_{1}\right) & \operatorname{Hom}\left(E_{2}, E_{1}\right) \\
\operatorname{Hom}\left(E_{1}, E_{2}\right) & \operatorname{End}\left(E_{2}\right)
\end{array}\right) .
$$

We will use the following description of the symmetric endomorphisms:

Lemma 3.1 Let $L_{0}$ be the principal polarization on $X$ induced by the principal polarizations on $E_{i}$. With respect to the Rosati-Involution associated with $L_{0}$, one has

$$
\operatorname{End}^{\operatorname{sym}}(X)=\left\{\left(\begin{array}{cc}
a & \widehat{\sigma} \\
\sigma & b
\end{array}\right) \mid a, b \in \mathbb{Z} \text { and } \sigma \in \operatorname{Hom}\left(E_{1}, E_{2}\right)\right\}
$$

(where $\widehat{\sigma}$ denotes the dual homomorphism of $\sigma$ ).

Proof. Let $\Lambda_{1}, \Lambda_{2} \subset \mathbb{C}$ be the lattices defining $E_{1}, E_{2}$. The hermitian form belonging to the principal polarization on $E_{i}$ is given by $H_{i}(x, y)=\frac{1}{a\left(\Lambda_{i}\right)} x \bar{y}$, where $a\left(\Lambda_{i}\right)$ 
denotes the area of a period parallelogram of the lattice $\Lambda_{i}$. For an endomorphism $\alpha=\left(\begin{array}{ll}\alpha_{11} & \alpha_{12} \\ \alpha_{21} & \alpha_{22}\end{array}\right) \in \operatorname{End}(X)$, the Rosati dual $\alpha^{\prime}$ is given by

$$
\alpha^{\prime}=\phi_{L_{0}}^{-1} \widehat{\alpha} \phi_{L_{0}}=\left(\begin{array}{cc}
\bar{\alpha}_{11} & \frac{a\left(\Lambda_{1}\right)}{a\left(\Lambda_{2}\right)} \cdot \bar{\alpha}_{21} \\
\frac{a\left(\Lambda_{2}\right)}{a\left(\Lambda_{1}\right)} \cdot \bar{\alpha}_{12} & \bar{\alpha}_{22}
\end{array}\right)=\left(\begin{array}{cc}
\bar{\alpha}_{11} & \widehat{\alpha}_{21} \\
\widehat{\alpha}_{12} & \bar{\alpha}_{22}
\end{array}\right) .
$$

The assertion then follows, since either $\operatorname{End}\left(E_{i}\right)=\mathbb{Z}$ or $\operatorname{End}\left(E_{i}\right)=\mathbb{Z}+\mathbb{Z} \omega_{i}$ for some $\omega_{i} \in \mathbb{C} \backslash \mathbb{R}$.

Type A: Products of non-isogenous elliptic curves. Suppose that $E_{1}$ and $E_{2}$ are not isogenous. We fix the principal polarization $L_{0}=\mathcal{O}_{X}\left(F_{1}+F_{2}\right)$, where $F_{1}$ and $F_{2}$ are the fibers of the projections. The fibers $F_{1}$ and $F_{2}$ are a basis of the lattice $\operatorname{NS}(X)$, with intersection matrix $\left(\begin{array}{ll}0 & 1 \\ 1 & 0\end{array}\right)$. So we have

$$
\Delta(X)=-1
$$

and therefore, with Proposition 1.1, we obtain for any ample $H$,

$$
\operatorname{Vol}(\operatorname{Nef}(X), H)=\frac{1}{\left(H^{2}\right)} .
$$

Type B: Products of isogenous elliptic curves without complex multiplication. Let $E_{1}, E_{2}$ be isogenous elliptic curves without complex multiplication. Let $\sigma: E_{1} \rightarrow E_{2}$ be an isogeny of minimal degree. We start by observing:

Lemma 3.2 We have

$$
\operatorname{Hom}\left(E_{1}, E_{2}\right)=\mathbb{Z} \cdot \sigma .
$$

Proof. Fix any isogeny $\alpha: E_{2} \rightarrow E_{1}$. The mapping $\operatorname{Hom}\left(E_{1}, E_{2}\right) \rightarrow \operatorname{End}\left(E_{1}\right)$, $\delta \mapsto \alpha \delta$, is an injective homomorphism of groups. Therefore $\operatorname{Hom}\left(E_{1}, E_{2}\right)$ is of rank 1. The assertion then follows from the minimality of $\sigma$.

From Lemma 3.1 we see that

$$
\operatorname{End}^{\mathrm{sym}}(X)=\left\{\left(\begin{array}{cc}
a & b \widehat{\sigma} \\
b \sigma & c
\end{array}\right) \mid a, b, c \in \mathbb{Z}\right\}
$$

As a consequence we get, upon using the isomorphism

$$
\varphi: \operatorname{NS}(X) \rightarrow \operatorname{End}^{\mathrm{sym}}(X), \quad L \mapsto \phi_{L_{0}}^{-1} \phi_{L},
$$

a basis $F_{1}, F_{2}, F_{3}$ of $\mathrm{NS}(X)$ with intersection matrix

$$
\left(\begin{array}{ccc}
0 & 1 & 0 \\
1 & 0 & 0 \\
0 & 0 & -2 \cdot \operatorname{deg}(\sigma)
\end{array}\right)
$$

So

$$
\Delta(X)=2 \cdot \operatorname{deg}(\sigma)
$$

and hence by Prop. 1.1 we get for any ample $H$,

$$
\operatorname{Vol}(\operatorname{Nef}(X), H)=\frac{\pi}{3 \cdot \sqrt{2 \cdot \operatorname{deg}(\sigma)} \cdot\left(H^{2}\right)^{\frac{3}{2}}} .
$$


Example 3.3 In the preceding formula every positive integer occurs as a minimal degree. In fact, consider for instance the elliptic curves $E_{1}$ and $E_{2}$ with $\Lambda_{1}=\mathbb{Z}+\mathbb{Z} \pi i$ and $\Lambda_{2}=\mathbb{Z}+\mathbb{Z} k \pi i$ for fixed $k \in \mathbb{N}$. Multiplication by $k$ gives an isogeny $E_{1} \rightarrow E_{2}$ of degree $k$, and there are no isogenies $E_{1} \rightarrow E_{2}$ of lower degree.

Remark 3.4 A self-product $E \times E$ of an elliptic curve without complex multiplication is of course a special case of the preceding considerations. However, this case can be dealt directly, without referring to the endomorphism ring: The classes of $F_{1}, F_{2}$ and the diagonal form a lattice basis of $\mathrm{NS}(X)$, and their intersection matrix is

$$
\left(\begin{array}{lll}
0 & 1 & 1 \\
1 & 0 & 1 \\
1 & 1 & 0
\end{array}\right)
$$

So we have $\Delta(X)=2$, and hence for any ample $H$,

$$
\operatorname{Vol}(\operatorname{Nef}(X), H)=\frac{\pi}{3 \cdot \sqrt{2} \cdot\left(H^{2}\right)^{\frac{3}{2}}} .
$$

Type C: Products of isogenous elliptic curves with complex multiplication. Suppose now that $E_{1}, E_{2}$ are isogenous and have complex multiplication, i.e., $\operatorname{End}_{\mathbb{Q}}\left(E_{i}\right)=\mathbb{Q}(\sqrt{d})$ for some square-free integer $d<0$. We have $\operatorname{End}\left(E_{i}\right)=\mathbb{Z}+f_{i} \omega \mathbb{Z}$, where $f_{1}, f_{2} \geqslant 1$ are integers and

$$
\omega=\left\{\begin{array}{ll}
\sqrt{d} & \text { if } d \equiv 2,3(\bmod 4) \\
\frac{1}{2}(1+\sqrt{d}) & \text { if } d \equiv 1(\bmod 4)
\end{array} .\right.
$$

The crucial result in this part is that one can determine the discriminant of the product $X=E_{1} \times E_{2}$ in terms of $\omega, f_{1}, f_{2}$, without explicitly referring to an isogeny:

Theorem 3.5 The discriminant of $\mathrm{NS}(X)$ is

$$
\Delta(X)=-4 \cdot \operatorname{LCM}\left(f_{1}, f_{2}\right)^{2} \cdot \operatorname{Im}(\omega)^{2} .
$$

With Prop. 1.1 we immediately conclude:

Corollary 3.6 We have for any ample $H$ :

$$
\operatorname{Vol}(\operatorname{Nef}(X), H)= \begin{cases}\frac{\pi}{6 \cdot \operatorname{LCM}\left(f_{1}, f_{2}\right) \sqrt{|d|}\left(H^{2}\right)^{2}} & \text { if } d \equiv 2,3(\bmod 4) \\ \frac{\pi}{3 \cdot \operatorname{LCM}\left(f_{1}, f_{2}\right) \sqrt{|d|}\left(H^{2}\right)^{2}} & \text { if } d \equiv 1(\bmod 4) .\end{cases}
$$

In the remainder of this section we will prove Theorem 3.5, We start by noting:

Lemma 3.7 The homomorphism group $\operatorname{Hom}\left(E_{1}, E_{2}\right)$ is a lattice in $\mathbb{C}$.

Proof. For any nonzero $\lambda \in \Lambda_{1}$, the mapping $\psi: \operatorname{Hom}\left(E_{1}, E_{2}\right) \rightarrow \Lambda_{2}, \sigma \mapsto \sigma \lambda$, is an injective homomorphism of groups. Therefore the rank of $\operatorname{Hom}\left(E_{1}, E_{2}\right)$ is at most 2, so it is in any event of the form $\operatorname{Hom}\left(E_{1}, E_{2}\right)=\mathbb{Z} \sigma_{1}+\mathbb{Z} \sigma_{2}$. We show that $\sigma_{1}$ and $\sigma_{2}$ are linear independent over $\mathbb{R}$ : Assume to the contrary that there is an $r \in \mathbb{R} \backslash\{0\}$ with $\sigma_{1}=r \sigma_{2}$. Then we find an $r^{\prime} \in \mathbb{R} \backslash\{0\}$ with $f_{2} \omega \sigma_{2}=r^{\prime} \sigma_{2}$, since $f_{2} \omega \sigma_{2}$ is a homomorphism $E_{1} \rightarrow E_{2}$. We conclude $f_{2} \omega=r^{\prime}$, which is a contradiction since $f_{2} \omega \in \mathbb{C} \backslash \mathbb{R}$. 
We fix now some $\mathbb{R}$-linear independent $\sigma_{1}, \sigma_{1} \in \mathbb{C}$ with $\operatorname{Hom}\left(E_{1}, E_{2}\right)=\mathbb{Z} \sigma_{1}+\mathbb{Z} \sigma_{2}$. Then we have

$$
\operatorname{Hom}\left(E_{2}, E_{1}\right)=\mathbb{Z} \widehat{\sigma}_{1}+\mathbb{Z} \widehat{\sigma}_{2} .
$$

with the dual isogenies $\widehat{\sigma}_{1}$ and $\widehat{\sigma}_{2}$. Via the isomorphism

$$
\varphi: \operatorname{NS}(X) \rightarrow \operatorname{End}^{\mathrm{sym}}(X), \quad L \mapsto \phi_{L_{0}}^{-1} \phi_{L}
$$

and

$$
\operatorname{End}^{\operatorname{sym}}(X)=\left\{\left(\begin{array}{cc}
a & b \widehat{\sigma}_{1}+c \widehat{\sigma}_{2} \\
b \sigma_{1}+c \sigma_{2} & d
\end{array}\right) \mid a, b, c, d \in \mathbb{Z}\right\}
$$

we get a basis $F_{1}, \ldots, F_{4}$ of $\mathrm{NS}(X)$ with intersection matrix

$$
\left(\begin{array}{cccc}
0 & 1 & 0 & 0 \\
1 & 0 & 0 & 0 \\
0 & 0 & -2 \sigma_{1} \widehat{\sigma}_{1} & -\left(\sigma_{1} \widehat{\sigma}_{2}+\sigma_{2} \widehat{\sigma}_{1}\right) \\
0 & 0 & -\left(\sigma_{1} \widehat{\sigma}_{2}+\sigma_{2} \widehat{\sigma}_{1}\right) & -2 \sigma_{2} \widehat{\sigma}_{2}
\end{array}\right) .
$$

The determinant of the matrix above is

$$
\Delta=\left(\sigma_{1} \widehat{\sigma}_{2}+\sigma_{2} \widehat{\sigma}_{1}\right)^{2}-4 \cdot \sigma_{1} \widehat{\sigma}_{1} \sigma_{2} \widehat{\sigma}_{2}=-4 \cdot \operatorname{Im}\left(\sigma_{1} \widehat{\sigma}_{2}\right)^{2} .
$$

Lemma 3.8 Let $E_{1}^{\prime}$ be an elliptic curve isomorphic to $E_{1}$ and $E_{2}^{\prime}$ an elliptic curve isomorphic to $E_{2}$. If $\left\{\delta_{1}, \delta_{2}\right\}$ is a basis of $\operatorname{Hom}\left(E_{1}^{\prime}, E_{2}^{\prime}\right)$, then we have

$$
\sigma_{1} \widehat{\sigma}_{2}=\delta_{1} \widehat{\delta_{2}}
$$

Proof. There are $c_{1}, c_{2} \in \mathbb{C}$ with $c_{i} \Lambda_{i}=\Lambda_{i}^{\prime}$. We have then

$$
\operatorname{Hom}\left(E_{1}^{\prime}, E_{2}^{\prime}\right)=\frac{c_{2}}{c_{1}} \operatorname{Hom}\left(E_{1}, E_{2}\right) \text {. }
$$

and therefore

$$
\delta_{1} \widehat{\delta_{2}}=\frac{c_{2}}{c_{1}} \sigma_{1} \widehat{\left(\frac{c_{2}}{c_{1}} \sigma_{2}\right)}=\frac{a\left(\Lambda_{1}^{\prime}\right)}{a\left(\Lambda_{2}^{\prime}\right)} \frac{\left|c_{2}\right|^{2}}{\left|c_{1}\right|^{2}} \sigma_{1} \bar{\sigma}_{2}=\frac{a\left(\Lambda_{1}\right)}{a\left(\Lambda_{2}\right)} \sigma_{1} \bar{\sigma}_{2}=\sigma_{1} \widehat{\sigma}_{2} .
$$

Proof of the theorem. After using a suitable isomorphism we can assume $\Lambda_{1} \subset \Lambda_{2}$. With the elementary divisor theorem we get

$$
\Lambda_{1}=\mathbb{Z} e_{1} u+\mathbb{Z} e_{2} v \subset \mathbb{Z} u+\mathbb{Z} v=\Lambda_{2}
$$

with $u, v \in \mathbb{C}$ and $e_{1}, e_{2} \in \mathbb{N}$ with $e_{1} \mid e_{2}$. Using again suitable isomorphisms we can assume (with Lemma 3.8) that

$$
\Lambda_{1}=\mathbb{Z}+\mathbb{Z} t \tau \subset \mathbb{Z}+\mathbb{Z} \tau=\Lambda_{2}
$$

with $t \in \mathbb{N}$ and $\tau \in \mathbb{C} \backslash \mathbb{R}$.

As above, we have $\operatorname{Hom}\left(E_{1}, E_{2}\right)=\mathbb{Z} \sigma_{1}+\mathbb{Z} \sigma_{2}$ for some $\sigma_{1}, \sigma_{2} \in \mathbb{C}$. Because of $\Lambda_{1} \subset \Lambda_{2}$, the identity is an element of $\operatorname{Hom}\left(E_{1}, E_{2}\right)$. Furthermore, $\mathbb{Z} \cdot 1$ is a primitive sublattice of $\operatorname{Hom}\left(E_{1}, E_{2}\right)$, since $\operatorname{Hom}\left(E_{1}, E_{2}\right)$ is a sublattice of $\Lambda_{2}$, and the latter 
contains no elements of $\mathbb{Q} \backslash \mathbb{Z}$. This shows that there is a basis of $\operatorname{Hom}\left(E_{1}, E_{2}\right)$ of the form $1, \sigma$. We fix such a basis for the remainder of the proof.

For any homomorphism $\lambda: E_{1} \rightarrow E_{2}$ we have

$$
\widehat{\lambda}=\frac{a\left(\Lambda_{1}\right)}{a\left(\Lambda_{2}\right)} \cdot \bar{\lambda}=\frac{|t \cdot \operatorname{Im}(\tau)|}{|\operatorname{Im}(\tau)|} \cdot \bar{\lambda}=t \cdot \bar{\lambda}
$$

Because of $1 \cdot \hat{\lambda} \in \operatorname{End}\left(E_{2}\right)$ and $\widehat{\lambda} \cdot 1 \in \operatorname{End}\left(E_{1}\right)$ we get

$$
\lambda \in \frac{1}{t} \cdot\left(\mathbb{Z}+\mathbb{Z} \cdot \operatorname{LCM}\left(f_{1}, f_{2}\right) \cdot \bar{\omega}\right) .
$$

So there is a $k \in \mathbb{Z}$ with

$$
\operatorname{Im}(\sigma)=k \cdot \frac{\operatorname{LCM}\left(f_{1}, f_{2}\right) \cdot \operatorname{Im}(\omega)}{t} .
$$

The number $\kappa:=\operatorname{LCM}\left(f_{1}, f_{2}\right) \cdot \omega$ defines an endomorphism of $E_{1}$, so $\kappa \cdot 1 \in \Lambda_{1}$, and hence there are integers $x, y$ with $\kappa=x+y \cdot t \tau$. Also, $\kappa$ defines an endomorphism of $E_{2}$, and hence $\kappa \tau \in \Lambda_{2}$. Now the number

$$
y \tau=\frac{-x+\kappa}{t}
$$

defines a homomorphism $E_{1} \rightarrow E_{2}$, because $y \tau \cdot 1 \in \Lambda_{2}$ and

$$
y \tau \cdot t \tau=\frac{-x+\kappa}{t} \cdot t \tau=-x \tau+\kappa \tau \in \Lambda_{2} .
$$

So we found that $\frac{-x+\kappa}{t} \in \mathbb{Z}+\mathbb{Z} \sigma$. Comparing now the imaginary part with $(*)$ we conclude that $k= \pm 1$. Therefore we obtain, using $(*)$,

$$
\left|\operatorname{Im}\left(\sigma_{1} \cdot \widehat{\sigma_{2}}\right)\right|=|\operatorname{Im}(1 \cdot \widehat{\sigma})|=|\operatorname{Im}(t \cdot \bar{\sigma})|=\operatorname{LCM}\left(f_{1}, f_{2}\right) \cdot|\operatorname{Im}(\omega)|,
$$

and this proves the theorem.

Remark 3.9 A self-product $E \times E$ of an elliptic curve with complex multiplication is a special case of Type C: In that situation we can take $\sigma_{1}=1$ and $\sigma_{2}=f \omega$ as generators of $\operatorname{Hom}\left(E_{1}, E_{2}\right)$, and get the discriminant

$$
\Delta(X)=\left\{\begin{array}{ll}
-4 f^{2}|d| & \text { if } d \equiv 2,3(\bmod 4) \\
-f^{2}|d| & \text { if } d \equiv 1(\bmod 4)
\end{array} .\right.
$$

\section{References}

[1] Bauer, Th., Schmitz, D.: Volumes of Zariski chambers. J. Pure Appl. Algebra 217(1), 153-164 (2013)

[2] Birkenhake, C., Lange, H.: Complex abelian varieties. Springer, 2004.

[3] Derenthal, U.: On a constant arising in Manin's conjecture for Del Pezzo surfaces. Math. Res. Lett. 3, 481-489 (2007)

[4] Derenthal, U.: Manin's conjecture for a quintic del Pezzo surface with A2 singularity. arXiv:0710.1583.

[5] Derenthal, U., Joyce, M., Teitler, Z.: The nef cone volume of generalized Del Pezzo surfaces. Algebra \& Number Theory 2, no. 2, pp. 157-182 (2008) 
[6] Derenthal, U., Browning, T.D.: Manin's conjecture for a cubic surface with D5 singularity. International Mathematics Research Notices IMRN (2009), no. 14, 2620-2647

[7] Derenthal, U., Browning, T. D.: Manin's conjecture for a quartic del Pezzo surface with A4 singularity. Annales de l'Institut Fourier 59 (2009), no. 3, 1231-1265

[8] Derenthal, U., Joyce, M., Teitler, Z.: The nef cone volume of generalized del Pezzo surfaces. Algebra \& Number Theory 2, 157-182 (2008)

[9] Peyre, E.: Hauteurs et mesures de Tamagawa sur les variétés de Fano. Duke Math. J. 79, 101-218 (1995)

[10] Runge, B.: Endomorphism rings of abelian surfaces and projective models of their moduli spaces. Tohoku Math. J. 51, 283-303 (1999)

Thomas Bauer, Fachbereich Mathematik und Informatik, Philipps-Universität Marburg, HansMeerwein-Straße, D-35032 Marburg, Germany.

E-mail address: tbauer@mathematik.uni-marburg.de

Carsten Bornträger Fachbereich Mathematik und Informatik, Philipps-Universität Marburg, Hans-Meerwein-Straße, D-35032 Marburg, Germany.

E-mail address: borntraegerc@mathematik.uni-marburg.de 